\title{
Características morfométricas de Talitroides topitotum (Burt) (Crustacea, Amphipoda, Talitridae) na Serra do Mar, Guaratuba, Paraná, Brasil ${ }^{1}$
}

\author{
Odete Lopez Lopes ${ }^{2} \&$ Setuko Masunari ${ }^{3}$ \\ ${ }^{1}$ Contribuição número 1419 do Departamento de Zoologia, Universidade Federal do Paraná. \\ ${ }^{2}$ Museu de História Natural Capão da Imbuia. Rua Benedito Conceição 407, 82810-080 Curitiba, Paraná, Brasil. E-mail: \\ odetellopes@yahoo.com.br \\ ${ }^{3}$ Departamento de Zoologia, Universidade Federal do Paraná. Caixa Postal 19020, 81531-980 Curitiba, Paraná, Brasil. E- \\ mail: setmas@ufpr.br
}

\begin{abstract}
Morphometric characteristics of Talitroides topitotum (Burt) (Crustacea, Amphipoda, Talitridae) from Serra do Mar, Guaratuba, Paraná, Brazil. A research on the relationship between the cephalic segment length (Lc) and total body length (Lt), and between the number of articles in the antenna flagellum and the total body length was conducted in a population of the terrestrial amphipod Talitroides topitotum (Burt, 1934) from Serra do Mar, Guaratuba, Paraná. It aimed to help the inference of the body length by measuring only the cephalic segment, and to know the relative age of individuals by counting the number of articles in their antenna flagellum. A total of 975 amphipods were caught with Malaise nets; they were photographed, the article number of the flagellum of the right and left antennae 1 and 2 was counted and cephalic segment length and their total body length was taken. There was a linear correlation between cephalic segment length and total body length defined through the equation Lc $=0.0506 \mathrm{Lt}+0.3297 ; \mathrm{r}^{2}=0.9577$. There was a significant difference in the article number between the right and left flagellum, for both pairs of antennae. The difference was at most four articles for the fist antenna pair and twelve articles for the second one. The highest number of these articles between right and left flagellum showed a linear correlation with the total body length classes and it was expressed by the equation $\mathrm{a}_{1}=4.3463 \mathrm{Lt}^{0.4264}$ for the first pair of antenna, and $\mathrm{a}_{2}=10.5700 \mathrm{Lt}^{0.3581}$ for the second pair. I was possible to infer the relative stage of development of $T$. topitotum by measuring the cephalic segment length. However, only the highest number of articles between right and left flagellum constitutes a reference parameter for the inference of individual relative age in this species.
\end{abstract}

KEY WORDS. Antenna articles, cephalic segment length, body length.

RESUMO. Um estudo sobre as relações morfométricas entre o comprimento cefálico (Lc) e o comprimento total do corpo (Lt), e o número de artículos do flagelo do primeiro e segundo pares de antenas e o comprimento total do corpo foi realizado em uma população de Talitroides topitotum (Burt, 1934), no entorno da Usina Hidroelétrica de Guaricana, Guaratuba, Paraná. O objetivo foi auxiliar na inferência do comprimento do corpo a partir do comprimento cefálico e da idade relativa dos exemplares pela contagem do número de artículos de seus flagelos antenais. Um total de 975 exemplares foi coletado e fotografado, dos quais os artículos dos flagelos direito e esquerdo das antenas 1 e 2 foram contados e os comprimentos do segmento cefálico e do corpo foram medidos. Houve uma correlação linear entre o comprimento do segmento cefálico e o comprimento total do corpo definido pela equação $L c=0,0506 \mathrm{Lt}+0,3297 ; \mathrm{r}^{2}=0,9577$. Diferenças significativas foram observadas entre os números de artículos dos flagelos direito e esquerdo de ambas as antenas. A diferença foi de até quatro artículos no primeiro par de antenas e de doze no segundo. $O$ maior número de artículos entre os dois flagelos mostrou uma correlação com a distribuição das classes de comprimento total do corpo com a equação $\mathrm{a}_{1}=4,3463 \mathrm{Lt}^{0,4264}$ para o primeiro par de antenas, e $\mathrm{a}_{2}=10,5700 \mathrm{Lt}^{0,3581}$ para o segundo par. Foi possível inferir o estágio relativo de desenvolvimento de $T$. topitotum pelas medidas do comprimento do segmento cefálico. Entretanto, somente o maior número de artículos entre os flagelos direito e esquerdo constitui parâmetro de referência para inferir a idade relativa dos indivíduos desta espécie.

PALAVRAS CHAVE. Artículos dos flagelos antenais, comprimento do segmento cefálico, comprimento do corpo. 
Talitroides topitotum (Burt, 1934) é um anfípodo terrestre da família Talitridae considerado endêmico em regiões tropicais e subtropicais na região indo-pacífica (Bousfield 1982, FrIEND \& RICHARDSON 1986); a espécie vem sendo introduzida em jardins, quintais e solos de floresta de vários países, dentre eles o Brasil (Bousfield 1960, Lemos de Castro 1972, Lemos de Castro \& PereiRA 1978, Ulian \& Mendes 1987). Fazem parte da fauna do solo e contribuem diretamente para o processo de decomposição da serapilheira (Ulian \& Mendes 1987, Richardson 1992) e para o aumento das taxas de oxigênio nesse ambiente (Friend \& RichARDSON 1986).

Poucos estudos de morfometria foram realizados com talitrídeos: LAM \& MA (1989) relacionaram, em T. topitotum, o comprimento cefálico com o comprimento total do corpo; PAGE (1979) analisou, em Orchestia traskiana Stimpson, 1857, o aumento no número de artículos no flagelo da segunda antena a cada ecdise, desde o estágio juvenil até o estágio adulto; Williams (1987) desenvolveu estudo semelhante ao de PAGE (1979) com Talitrus saltator (Montagu, 1808); O'HANlon \& Bolger (1997) relacionaram, em Arcitalitrus dorrieni (Hunt, 1925), o número de artículos do flagelo da segunda antena com o comprimento total; Lindeman (1991) relacionou em Cerrorchestia hyloraina Lindeman, 1991, o número de artículos do flagelo da primeira e da segunda antena do lado direito com o comprimento total do corpo. Em todos estes estudos houve correlação entre as medidas analisadas.

Na Serra do Mar paranaense, uma população de $T$. topitotum ocorre em abundância na área de entorno da Usina Hidroelétrica de Guaricana, em Guaratuba, onde a umidade relativa do ar oscila de $72,75 \%$ a $93,07 \%$ e a temperatura do ar varia de $9,0^{\circ} \mathrm{C}$ a $37,5^{\circ} \mathrm{C}$ durante o ano, a uma altitude de $270 \mathrm{~m}$ (Lopes \& MASUnARi 2004). A literatura não contempla qualquer estudo morfométrico em anfípodos terrestres ocorrentes nos ecossistemas brasileiros.

O objetivo deste trabalho consite em analisar as relações entre o comprimento cefálico e o número de artículos do flagelo dos dois pares de antenas com o comprimento total do corpo, bem como a variabilidade do número de artículos do flagelo das antenas na referida população de T. topitotum ocorrente em Guaricana, na Serra do Mar, visando contribuir com o conhecimento das características morfométricas desta espécie.

\section{MATERIAL E MÉTODOS}

Amostras de T. topitotum correspondendo a um mês em cada estação do ano foram obtidas nos períodos de 22 a 26 de julho de 1985, 21 a 25 de outubro de 1985, 27 a 31 de janeiro de 1986 e 28 de abril de 1986 a 02 de maio 1986. Foram utilizadas Redes de Malaise em oito estações de coleta, localizadas na Serra do Mar paranaense. Quatro das estações foram estabelecidas à altitude de $270 \mathrm{~m}$, na área próxima à Usina Hidroelética de Guaricana, no município de Guaratuba, e outras quatro à $720 \mathrm{~m}$ de altitude, na área de entorno da represa de mesmo nome, no município de São José dos Pinhais. Entre- tanto, todas as amostras foram consideradas como pertencentes a uma única população de T. topitotum, comprovada através da verificação da inclusão de $95 \%$ dos dados de comprimento cefálico e do comprimento total do corpo, dentro do intervalo de confiança de 5\%. Uma descrição mais detalhada da área de estudo e dos métodos de coleta utilizados pode ser consultada em Lopes \& MASUNARI (2004).

Os animais foram fotografados em decúbito lateral direito, com câmera digital instalada em microscópio estereoscópico, utilizando o programa para microcomputadores ASUS digital VCR. Durante a captura das imagens, foram contados os artículos dos flagelos direito e esquerdo do primeiro e segundo pares de antenas ( $\mathrm{a}_{1}$ e $\mathrm{a}_{2}$, respectivamente). Como não ocorreram machos neste estudo, não houve necessidade de analisar exemplares agrupados por sexo.

As imagens digitais foram medidas utilizando o programa Jandel Sigma Scan ${ }^{\circledR}$ Pro Image Measurement, versão 2.0, com precisão de centésimo de milímetro. De cada exemplar foram medidos o comprimento cefálico (Lc), a partir da inserção das antenas até a margem posterior do mesmo e o comprimento dorsal de cada somito torácico e abdominal incluindo o telson. O comprimento total do corpo (Lt) foi obtido pela somatória dos valores do comprimento cefálico e dos comprimentos dos somitos torácicos e abdominais.

O número de classes de comprimento total do corpo foi determinado pela regra de Sturge (VIEIRA 1980) através da fórmula: $\mathrm{nc}=1+[\log (\mathrm{n}) / \log (2)]$, sendo "nc" o número de classes e " $n$ " o número de exemplares analisados. O intervalo de cada classe foi definido segundo Siegel (1956) por Ic $=\lambda / \mathrm{nc}$, onde "Ic" é o intervalo da classe, "nc" o número de classes e " $\lambda$ " a amplitude de variação de comprimento. As classes obtidas foram testadas pelo teste de Kolmogorov-Smirnov (SIEgel 1956) para a comprovação da normalidade.

As equações das retas dos dados de comprimento cefálico e total do corpo foram obtidas por regressão linear. No estudo da relação entre o número de artículos dos flagelos de $\mathrm{a}_{1}$ e $\mathrm{a}_{2} \mathrm{e}$ $\mathrm{o}$ Lt, foi escolhido o número maior entre as antenas direita $\mathrm{e}$ esquerda, já que houve diferença no número de artículos nos flagelos do mesmo par de antenas. As equações lineares foram obtidas pelo método dos mínimos quadrados com os valores de comprimento total do corpo e de número de artículos logaritmizados. Os exemplares com antenas quebradas foram excluídos desta análise. A significância da diferença entre o número de artículos do flagelo de a1 e a2 foi testada pelo " $\mathrm{t}$ " crítico de Student $(\alpha=0,05)$, aplicado sobre a mediana dos valores registrados.

\section{RESULTADOS}

Foram analisados 975 exemplares, todos fêmeas adultas, sendo 274 ovígeras e 701 não ovígeras. O comprimento total do corpo variou de 6,02 a 13,51 mm nos exemplares amostrados a $270 \mathrm{~m}$ de altitude e de 5,74 a 13,69 mm naqueles amostrados a $720 \mathrm{~m}$. 
O comprimento total do corpo foi distribuído em classes de $0,7 \mathrm{~mm}$ de amplitude. A relação entre as classes de comprimento do segmento cefálico e as classes de comprimento total do corpo foi de $\mathrm{Lc}=0,3449+0,0506 \mathrm{Lt}\left(\mathrm{n}=975 ; \mathrm{r}^{2}=0,96 ; \mathrm{F}=\right.$ 173,30; $<$ <,05) na população inteira, incluindo as amostras de $270 \mathrm{~m}$ e $720 \mathrm{~m}$ de altitude (Fig. 1).

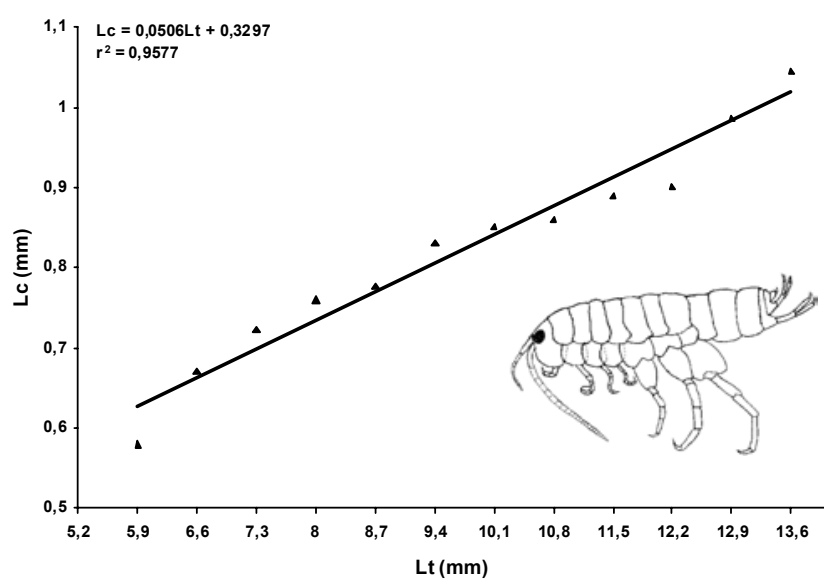

Figura 1. Talitroides topitotum. Dispersão entre as médias das medidas de comprimento do segmento cefálico (LC) e do comprimento total do corpo (Lt) de fêmeas adultas para cada classe de comprimento. Estão representados os pontos médios das classes de comprimento, $\mathrm{n}=975$.

A equação Lc $=0,2469+0,0625 \mathrm{Lt}\left(\mathrm{n}=384 ; \mathrm{r}^{2}=0,87 ; \mathrm{p}<\right.$ $0,05)$ relativa aos dados amostrados a $270 \mathrm{~m}$ de altitude e a equação Lc $=0,3797+0,0463 \mathrm{Lt}\left(\mathrm{n}=591 ; \mathrm{r}^{2}=0,97 ; \mathrm{p}<0,05\right)$ relativa aos amostrados a $720 \mathrm{~m}$ foram comparadas com a equação $\mathrm{Lc}=0,3449+0,0506 \mathrm{Lt}$ relativa à população total acrescida do intervalo de confiança (Ic $= \pm 0,0679$ ). Este procedimento permitiu vizualizar que houve inclusão de $95,24 \%$ de todos os dados plotados para as duas altitudes dentro do intervalo de confiança, e portanto, trata-se de uma única população possuindo as mesmas características morfométricas independentemente da altitude e da estação do ano, nas quais as amostras foram obtidas.

$\mathrm{Na}$ busca de relações entre o número de artículos dos flagelos de $\mathrm{a}_{1}$ e $\mathrm{a}_{2}$ com Lt verificou-se quantidades diferentes de artículos no flagelo da antena direita e esquerda, tanto em $\mathrm{a}_{1}$ como em $\mathrm{a}_{2}$. Foi analisada a significância desta diferença (Figs 2 e 3$)$.

O número de artículos do flagelo variou em $\mathrm{a}_{1}$ de oito a 13 tanto no direito quanto no esquerdo em 733 anfípodos analisados; em $\mathrm{a}_{2}$, houve de 10 a 31 artículos no esquerdo, e de 10 a 30 no direito em 772 anfípodos. A amplitude de variação da diferença no número de artículos do flagelo nas classes de comprimento do corpo variou de zero a quatro artículos em $\mathrm{a}_{1}$ en-
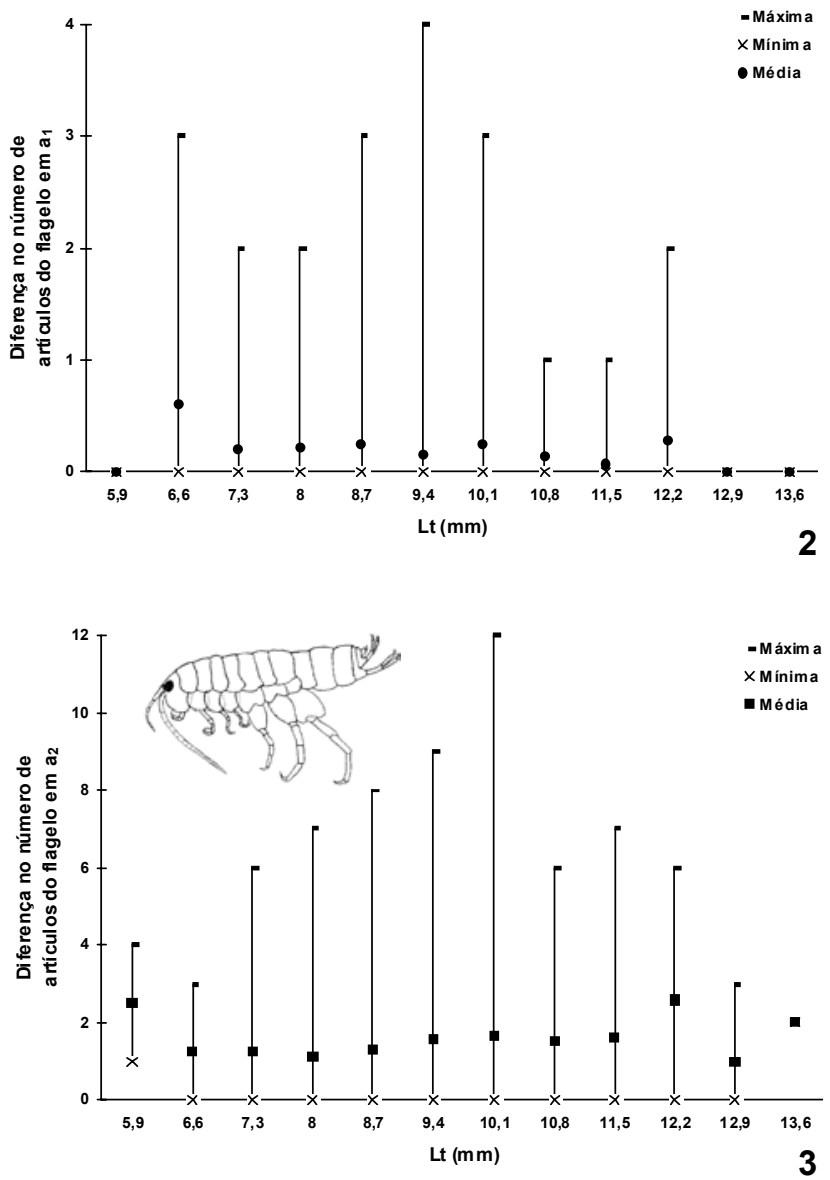

Figuras 2-3. Talitroides topitotum. Distribuição da diferença no número de artículos dos flagelos direito e esquerdo do primeiro par de antenas " $\mathrm{a}_{1}$ ", $\mathrm{n}=733$ (2) e " $\mathrm{a}_{2}{ }^{\prime}, \mathrm{n}=772$ ( 3). Os pontos indicam a média e as extremidades das linhas verticais, os valores máximos e mínimos para cada classe de comprimento total do corpo (Lt). Estão indicados os pontos médios de cada classe.

quanto em $a_{2}$ foi de um a doze artículos. Não houve correlação entre a amplitude de variação da diferença no número de artículos entre os dois flagelos e as classes de comprimento total do corpo: anfípodos pequenos tiveram amplitudes pequenas e grandes e vice-versa, tanto em $\mathrm{a}_{1}$ como em $\mathrm{a}_{2}$. Dos 733 anfípodos analisados para $a_{1}$ e 772 para $a_{2}$, somente $93,04 \%$ e $80,70 \%$, respectivamente, ficaram incluídos no intervalo de confiança da diferença entre os flagelos direito e esquerdo (Tabs I e II). As diferenças entre os números de artículos dos flagelos direito e esquerdo de $\mathrm{a}_{1}$ e $\mathrm{a}_{2}$ foram, portanto, consideradas significativas.

A relação entre o número máximo de artículos do flagelo e o comprimento total do corpo em cada classe foi de $\mathrm{a}_{1}=$ $4,3463 \mathrm{Lt}^{\mathrm{o}, 4264}\left(\mathrm{n}=733 ; \mathrm{r}^{2}=0,98 ; \mathrm{F}=444,38 ; \mathrm{p}<0,05\right)$, para $\mathrm{a}_{1}$ (Fig. 4) e $\mathrm{a}_{2}=10,5700 \mathrm{Lt}^{\mathrm{o}, 3581}\left(\mathrm{n}=772 ; \mathrm{r}^{2}=0,95 ; \mathrm{F}=40,96 ; \mathrm{p}<\right.$ 0,05), para $a_{2}$ (Fig. 5). 

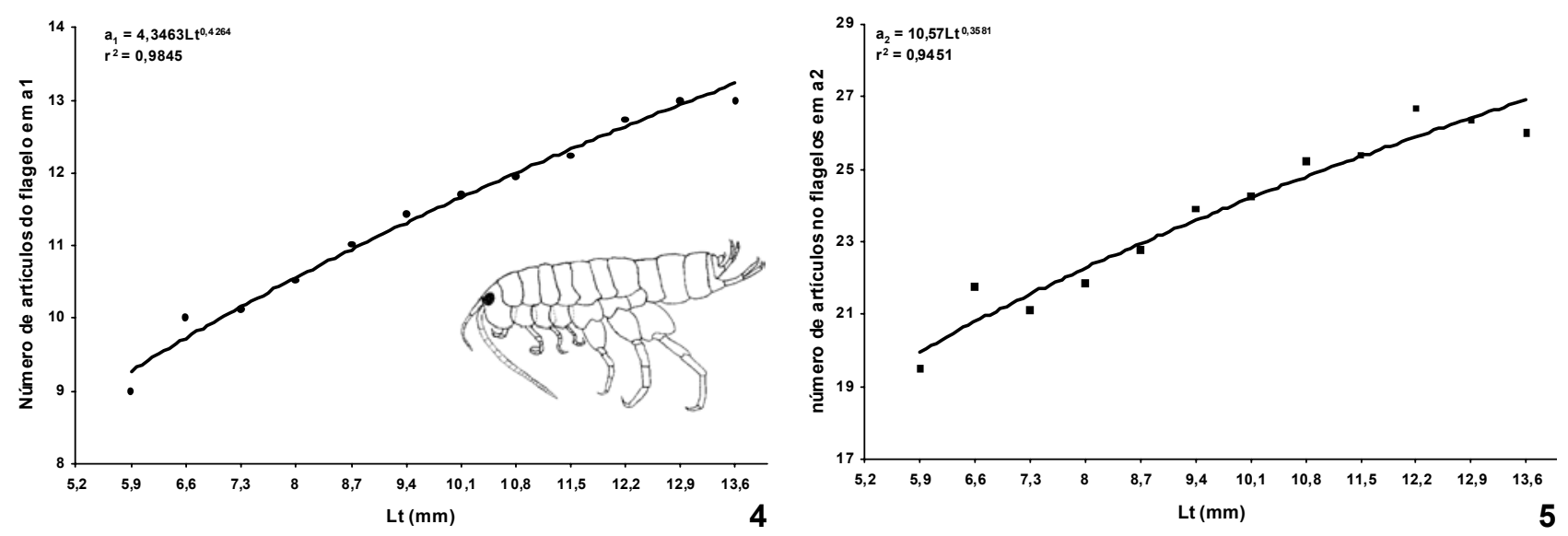

Figuras 4-5. Talitroides topitotum. Distribuição do maior número de artículos nos flagelos do primeiro par de antenas " $\mathrm{a}_{1}{ }^{\prime}, \mathrm{n}=733$ (4) e " $\mathrm{a}_{2}{ }^{\prime}, \mathrm{n}=772$ (5), nas classes de comprimento total do corpo (Lt). Estão indicados os pontos médios das classes de comprimento, $\mathrm{n}=733$.

\section{DISCUSSÃO}

Os resultados do presente trabalho corroboram o de LAM \& MA (1989) que encontraram, também, uma relação linear entre o comprimento cefálico e o comprimento total do corpo em $T$. topitotum ( $\mathrm{Lt}=-1,39+9,94 \mathrm{Lc} ; \mathrm{r}=0,99 ; \mathrm{n}=50 ; \mathrm{p}<0,001$ ) ocorrente em Hong Kong. A metodologia de obtenção de dados morfométricos adotada por estes autores difere daquela utilizada no presente trabalho no que concerne à posição dos exemplares durante a mensuração e ao instrumento de medida de comprimento utilizado: a região dorsal do animal endireitada até assumir a posição retilínea, foi medida com uma ocular milimetrada em microscópio estereoscópico. Entretanto, esta diferença na metodologia não anulou a possibilidade de obter uma regressão linear entre estas duas medidas em ambas as populações de T. topitotum. O coeficiente de correlação de Pearson $(\mathrm{r}=0,98)$ obtido no presente trabalho foi um pouco menor do que o registrado para a população de Hong Kong $(r=0,99)$ o que pode estar relacionado com o maior número de exemplares analisados na população de Guaricana ( $\mathrm{n}=975$ contra $\mathrm{n}=50$ de Hong Kong) ou de uma leve deformação dos segmentos do corpo em função do longo tempo de fixação (15 anos).

Pela primeira vez registrou-se uma diferença estatisticamente significativa no número de artículos dos flagelos direito e esquerdo para ambos os pares de antenas. Em estudos morfométricos com machos de Cerrorchestia hyloraina, LindemAN (1991) levou em consideração os artículos somente do flagelo direito de $\mathrm{a}_{1}$ e $\mathrm{a}_{2}$ e a equação obtida foi $\left(\mathrm{a}_{1}+\mathrm{a}_{2}\right)=1,34+0,18 \mathrm{Lt}$ $\left(\mathrm{n}=372, \mathrm{r}^{2}=0,94\right)$. A autora relata ainda, que as fêmeas apresentaram sempre dois artículos a menos no flagelo em comparação com os machos, porém, não há informações sobre uma possível diferença no número de artículos entre as antenas direita e esquerda, para nenhum dos sexos.
Fêmeas adultas de Arcitalitrus dorrieni apresentam, entre o número de artículos do flagelo da segunda antena e o comprimento total do corpo, relação expressa pela equação $\mathrm{a}_{2}=$ $-1,97+3,67 \mathrm{Lt}-0,10(\mathrm{Lt})^{2}(\mathrm{n}=289, \mathrm{~F}=1520,77, \mathrm{p}<0,0001)$ para os exemplares amostrados em Addergoole (Irlanda) e $\mathrm{a}_{2}=$ $-2,17+3,62 \mathrm{Lt}-0,10(\mathrm{Lt})^{2}(\mathrm{n}=489 ; \mathrm{F}=3013,71 ; \mathrm{p}=0,0001)$ para os amostrados em Tullywee Bridge (Irlanda) (O'HANLon \& Bolger 1997). Portanto, para cada população de uma mesma espécie de talitrídeo, há evidências que relações diferentes no aumento de artículos a cada ecdise. Infelizmente, estes autores também não fornecem informações sobre uma possível diferença no número de artículos entre as antenas direita e esquerda.

As equações obtidas no presente trabalho permitem concluir que o número de artículos do flagelo tende a aumentar com o crescimento do indivíduo em T. topitotum. Apesar do elevado coeficiente de determinação obtido para ambas as equações, $98,45 \%$ para $a_{1}$ e $94,51 \%$ para $a_{2}$, as equações das figuras 4 e 5 não permitem uma avaliação precisa do número de artículos correspondentes a cada comprimento do corpo, para fins de estimativa da idade dos anfípodos. Um mesmo número de artículos pode corresponder a uma faixa bastante ampla de comprimento total do corpo. Dez artículos na flagelo de $a_{1}$ correspondem a um comprimento total do corpo que varia de $6,30 \mathrm{~mm}$ a 11,99 $\mathrm{mm}$, por exemplo (Tabs I e II). A mesma análise é possivel para $a_{2}$. PAge (1979), comparando dados de observações em campo e de experimentos em laboratório com Orchestia traskiana, acompanhou o incremento de artículos no flagelo de $\mathrm{a}_{2}$ a cada ecdise; Williams (1987) realizou estudo semelhante com Talitrus saltator. Ambos concluíram que o aumento de artículos pode variar de zero a dois artículos a cada muda. A dificuldade em estabelecer uma relação simples que indique o crescimento de cada indivíduo e que permita conclusões mais claras, evidencia a necessidade de novos estudos.

Revista Brasileira de Zoologia 21 (4): 779-784, dezembro 2004 
Tabela I. Talitroides topitotum. Números máximo e mínimo de artículos do flagelo direito e esquerdo do primeiro par de antenas (a1), comparados com o intervalo de confiança, em cada classe de comprimento total do corpo (Lt). (*) Valores excluídos do intervalo de confiança. Estão indicados os pontos médios das classes de comprimento, $\mathrm{n}=733$.

\begin{tabular}{|c|c|c|c|c|c|c|}
\hline \multirow{2}{*}{ Lt (mm) } & \multicolumn{2}{|c|}{$\begin{array}{l}\text { Número de artículos no flagelo } \\
\text { direito da primeira antena }\end{array}$} & \multicolumn{2}{|c|}{ Intervalo de confiança $(\mathrm{Ic}=1,36)$} & \multicolumn{2}{|c|}{$\begin{array}{l}\text { Número de artículos no flagelo } \\
\text { esquerdo da primeira antena }\end{array}$} \\
\hline & Mínimo & Máximo & Mínimo & Máximo & Mínimo & Máximo \\
\hline $5,60-6,29$ & 9 & 9 & 7,64 & 10,36 & 9 & 9 \\
\hline $6,30-6,99$ & 9 & $13^{*}$ & 8,54 & 11,26 & 9 & 10 \\
\hline $7,00-7,69$ & 8* & 11 & 8,65 & 11,37 & 8* & 11 \\
\hline $7,70-8,39$ & 9 & 12 & 9,04 & 11,76 & $8^{*}$ & 12 \\
\hline $8,40-9,09$ & 9 & 12 & 9,52 & 12,24 & 8* & 12 \\
\hline $9,10-9,79$ & 8* & 13 & 9,99 & 12,71 & $8^{*}$ & 13 \\
\hline $9,80-10,49$ & $8^{*}$ & 13 & 10,22 & 12,94 & $8^{*}$ & 13 \\
\hline $10,50-11,19$ & 9* & 13 & 10,52 & 13,24 & 9* & 13 \\
\hline $11,20-11,89$ & 11 & 13 & 10,85 & 13,57 & 11 & 13 \\
\hline $11,90-12,59$ & 11 & 13 & 11,23 & 13,95 & 12 & 13 \\
\hline $12,60-13,29$ & 13 & 13 & 11,64 & 14,36 & 13 & 13 \\
\hline $13,30-13,99$ & 13 & 13 & 11,64 & 14,36 & 13 & 13 \\
\hline \multicolumn{5}{|c|}{ Exemplares incluídos no intervalo de confiança } & & 682 \\
\hline \multicolumn{5}{|c|}{ Percentual de inclusão (\%) } & & 93,04 \\
\hline
\end{tabular}

Tabela II. Talitroides topitotum. Números máximo e mínimo de artículos do flagelo direito e esquerdo do segundo par de antenas (a2), comparados com o intervalo de confiança, em cada classe de comprimento total do corpo (Lt). (*) Valores excluídos do intervalo de confiança. Estão indicados os pontos médios das classes de comprimento, $\mathrm{n}=772$.

\begin{tabular}{|c|c|c|c|c|c|c|}
\hline \multirow[t]{2}{*}{ Lt (mm) } & \multicolumn{2}{|c|}{$\begin{array}{l}\text { Número de artículos no flagelo direito } \\
\text { da segunda antena }\end{array}$} & \multicolumn{2}{|c|}{ Intervalo de confiança $(\mathrm{Ic}=3,40)$} & \multicolumn{2}{|c|}{$\begin{array}{l}\text { Número de artículos no flagelo } \\
\text { esquerdo da segunda antena }\end{array}$} \\
\hline & mínimo & máximo & mínimo & máximo & mínimo & máximo \\
\hline $5,60-6,29$ & 19 & 20 & 14,85 & 21,65 & 15 & 19 \\
\hline $6,30-6,99$ & 21 & 23 & 17,73 & 24,53 & 18 & 22 \\
\hline $7,00-7,69$ & $16^{*}$ & 23 & 17,08 & 23,88 & $15^{*}$ & 23 \\
\hline $7,70-8,39$ & $10 *$ & $26 *$ & 17,88 & 24,68 & $10 *$ & 25 \\
\hline $8,40-9,09$ & $14^{*}$ & 26 & 18,74 & 25,54 & $12^{*}$ & $27 *$ \\
\hline $9,10-9,79$ & $15^{*}$ & $29 *$ & 19,71 & 26,51 & $16^{*}$ & $28^{*}$ \\
\hline $9,80-10,49$ & $12^{*}$ & 27 & 19,99 & 26,79 & $14^{*}$ & $28 *$ \\
\hline $10,50-11,19$ & $18 *$ & 28 & 21,05 & 27,85 & $18^{*}$ & 29* \\
\hline $11,20-11,89$ & 21 & 28 & 21,17 & 27,97 & $18^{*}$ & 28 \\
\hline $11,90-12,59$ & 22 & 28 & 21,98 & 28,78 & $19 *$ & $30 *$ \\
\hline $12,60-13,29$ & 24 & $31 *$ & 22,43 & 29,23 & 24 & 28 \\
\hline $13,30-13,99$ & 24 & 24 & 21,60 & 28,40 & 26 & 26 \\
\hline \multicolumn{5}{|c|}{ Exemplares incluídos no intervalo de confiança } & & 623 \\
\hline \multicolumn{3}{|c|}{ Percentual de inclusão (\%) } & & & & 80,70 \\
\hline
\end{tabular}




\section{AGRADECIMENTOS}

Ao CNPq pela bolsa de Mestrado conferida à primeira autora. Aos coordenadores do Curso de Pós-graduação em Zoologia da UFPR pela possibilidade do desenvolvimento da dissertação de Mestrado, que originou o presente artigo.

\section{REFERÊNCIAS BIBLIOGRÁFICAS}

Bousfield, E.L. 1960. New records of beach hoppers (Crustacea: Amphipoda) from the coast of California. Bulletin of National Museum of Canada, Ottawa, 172: 1-12.

. 1982. The amphipod Superfamily Talitroidea in the Northeastern Pacific region. I. Family Talitridae: systematics and distributional ecology. National Museum of Natural Sciences Publications in Biological Oceanography, Otawa, 11: 1-73.

Friend, J.A. \& A.M.M. Richardson. 1986. Biology of terrestrial amphipods. Annual Review of Entomology, Palo Alto, 31: 25-48.

LAM, P.K.S. \& H.H.T. MA. 1989. Some observations on the cycle and population dynamics of Talitroides topitotum (Burt) (Amphipoda; Talitridae) in Hong Kong. Journal of Natural History, London, 23: 1087-1092.

Lemos de Castro, A.L. 1972. Talitrus (Talitroides) pacificus Hurley, anfípodo terrestre introduzido em São Paulo. Arquivos do Instituto Biológico São Paulo, São Paulo, 39 (3): 201-203.

Lemos de Castro, A.L. \& V.F.G. Pereira. 1978. Anfípodos terrestres do gênero Talitrus introduzidos no Brasil (Amphipoda, Talitridae). Atas da Sociedade Biológica do Rio de Janeiro, Rio de Janeiro, 19: 47-49.

LINDEMAN, D. 1991. Natural history of the terrestrial amphipod Cerrorchestia hyloraina Lindeman (Crustacea: Amphipoda;
Talitridae) in a Costa Rican cloud forest. Journal of Natural History, London, 25: 623-638.

Lopes, O.L. \& S. Masunari. 2004. Distribuição de abundância de Talitroides topitotum (Burt) (Crustacea, Amphipoda, Talitridae) na área de entorno da Usina Hidroelétrica de Guaricana, Serra do Mar, Guaratuba, Paraná, Brasil. Revista Brasileira de Zoologia, Curitiba, 21 (2): 219-227.

O'Hanlon, R.P. \& T. Bolger. 1997. Biomass, growth, and secundary production of Arcitalitrus dorrieni (Crustacea: Amphipoda: Talitridae) at two sites in Co. Galway, Ireland. Journal of zoology, London, 241: 409-428.

PAGE, H.M. 1979. Relationship between growth, size, molting, and number of antennal segments in Orchestia traskiana Stimpson (Amphipoda, Talitridae). Crustaceana, Leiden, 37 (3): 247-252.

RICHARDSON, A.M.M. 1992. Altitudinal distribution of native and alien landhoppers (Amphipoda: Talitridae) in the Ko'olau Range, O'ahu, Hawaiian Islands. Journal of Natural History, London, 26: 339-352.

SIEGEL, S. 1956. Estatística não paramétrica (para as ciências do comportamento). Rio de Janeiro, McGraw-Hill, XIX+350p.

Ulian, G.B. \& E.G. Mendes. 1987. Preferences of a terrestrial amphipod, Talitrus (Talitroides) pacificus, Hurley, 1955, towards some environmental factors. Revista Brasileira de Biologia, Rio de Janeiro, 47 (3): 247-256.

VIEIRA, S. 1980. Introdução à bioestatística. Rio de Janeiro, Campus, $3^{\text {a }}$ ed., XVIII+196p.

Williams, J.A. 1987. The relationship between antennal segment number and moulting in Talitrus saltator (Montagu, 1808) (Amphipoda, Talitridae). Crustaceana, Leiden, 53 (3): 243252.

Recebido em 30.V.2003; aceito em 27.IX.2004.

Revista Brasileira de Zoologia 21 (4): 779-784, dezembro 2004 